\title{
Complete Response to PD-I Inhibitor in Primary Hepatocellular Carcinoma Patients Post-Progression on Bi-Specific Antibody Conjugated CIK Cell Treatment: A Report of Two Cases
}

Tong $\mathrm{Wu}^{*}$

Linzhi Zhang*

Zhen Zeng

Tao Yan

Jiamin Cheng

Xiaojie Miao

Yinying Lu

Comprehensive Liver Cancer Center, The 5th Medicine Center of PLA General Hospital, Beijing, 100039, People's

Republic of China

*These authors contributed equally to this work
Background: Programmed death receptor-1 (PD-1) immune checkpoint inhibitors (ICIs) have produced encouraging results in hepatocellular carcinoma (HCC) patients. Cytokineinduced killer (CIK) cells treatment can specifically identify tumor-associated antigens and has encouraging preliminary efficacy for HCC. This study reported two cases of HCC patients achieved complete response (CR) by anti-PD-1 antibody therapy post-progression on bi-specific antibody conjugated CIK immunotherapy.

Case Presentation: Case one, a 75-year-old male, was diagnosed with the intrahepatic cholangiocarcinoma (ICC) in October 2017. After interventional, CIK, ablation and other comprehensive therapy, ICC was gradually cured. When new occurrence of $\mathrm{HCC}$, he was treated with anti-PD-1 antibody therapy and achieved CR. Case two, a 65-year-old female, was diagnosed with HCC in July 2016. After progression on several ablation treatments, she received 8 cycles of CIK treatment and achieved stable disease (SD). After disease progressed on CIK treatment, she received 4 cycles of anti-PD-1 antibody therapy, finally achieved CR.

Conclusion: Anti-PD-1 antibody therapy after prior progression on bi-specific antibody conjugated CIK immunotherapy may be efficacy and safety for HCC patients.

Keywords: cytokine-induced killer, programmed cell death-1, hepatocellular carcinoma, complete response, liver

\section{Introduction}

Recently, the emergence of immune checkpoint inhibitors (ICIs) has greatly improved the prognosis of patients with hepatocellular carcinoma (HCC), ${ }^{1}$ such as atezolizumab combined with bevacizumab, ${ }^{2}$ pembrolizumab combined with lenvatinib, ${ }^{3}$ and ipilimumab (a fully human monoclonal antibody cytotoxic T-lymphocyte antigen 4 (CTLA-4)) combined with nivolumab (an antiprogrammed cell death-1, anti-PD-1). ${ }^{4}$ Tislelizumab and Sintilimab, as anti-PD-1 monoclonal antibodies, are being evaluated as an immunotherapeutic for patients with advanced HCC. ${ }^{5,6}$ Cytokine-induced killer cells (CIK), as the most commonly used cell-based immunotherapy, display cytolytic activity against a wide range of cancer cells and have encouraging preliminary efficacy for HCC. ${ }^{7,8}$ Recently, some studies have reported that CIK combined with anti-PD-1 antibody therapy could offer a pragmatic treatment option for patients with metastatic renal carcinoma and non-small cell lung cancer. ${ }^{9,10}$
Correspondence: Yinying Lu

Tel +8613301256799

Email luyinying1973@163.com 
However, anti-PD-1 antibody therapy after progression on CIK in HCC patients has not been reported. It may be the idea of future clinical research to explore new therapeutic methods and realize the individualization and precision of HCC treatment. Here, the present study reported two cases of HCC patients achieved complete response (CR) by anti-PD-1 antibody therapy after bi-specific antibody conjugated CIK immunotherapy failure.

\section{Case Presentation Clinical Case One}

A 75-year-old male, who had medical history of hepatitis $\mathrm{B}$, coronary heart disease and atrial fibrillation (AF), was admitted to our hospital due to abdominal distension in October 2017. The contrast-enhanced abdominal magnetic resonance imaging (MRI) showed multiple hepatic masses with partial fusion, which was considered to be HCC, Child-Pugh grade A (5 points). The result of HBV-DNA test was $6.25 \times 10^{\wedge} 5 \mathrm{IU} / \mathrm{mL}$, serum $\alpha$-fetoprotein (AFP) level was normal and serum CA199 was $52.26 \mathrm{ng} / \mathrm{mL}$. The pathological results of liver biopsy showed intrahepatic cholangiocarcinoma (ICC), poorly differentiated, MUC-1 positive (+) (Figure 1A). Finally, he was histologically confirmed with T2N0M0 stage II primary HCC, compensated hepatitis B virus (HBV) cirrhosis, arrhyth$\mathrm{mia} / \mathrm{AF}$.
In November 2017 and January 2018, he received transarterial chemoembolization (TACE) treatment, during that time he was induced severe AF after 2 cycles (6 weeks) of regorafenib therapy and discontinued it. In March 2018, the abdominal $\mathrm{CT}$ showed he had increased residual activity of lesion in liver S6 and new active lesions in liver S5, and little change of remaining lesions, and the efficacy was assessed as progressive disease (PD, Supplementary Figure 1). Then, this case was enrolled in the clinical trial "Phase II Randomized, Controlled Clinical Study of Activated CIK Armed with Bispecific Antibody for Advanced Liver Cancer" (NTC03146637), and was treated with bi-specific antibody conjugated CIK cells (MUC-1) for 4 cycles (Supplementary Figure 2). On August 19, 2018, the CA199 reexamination was normal, the efficacy was assessed as stable disease (SD) or partial response (PR) according to response evaluation criteria in solid tumors (RECIST) or modified RECIST, and tumor activity was significantly reduced.

In October 2018, abdominal MRI showed liver S5 and S6 lesions were significantly larger than before (Supplementary Figure 3A), and non-target lesions continued to necrotic absorption. AFP level increased to $245.3 \mathrm{ng} /$ $\mathrm{mL}$ (Supplementary Figure 3B). Liver S5 and S6 lesions were performed with ultrasound-guided microwave ablation and liver biopsy. Postoperative pathology showed the combined HCC and ICC (cHCC-ICC), moderately differentiated (Figure 1B). Immunohistochemical results showed that

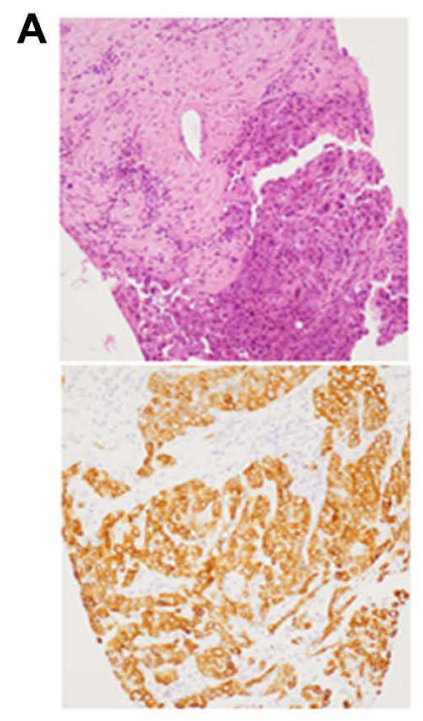

\section{B}

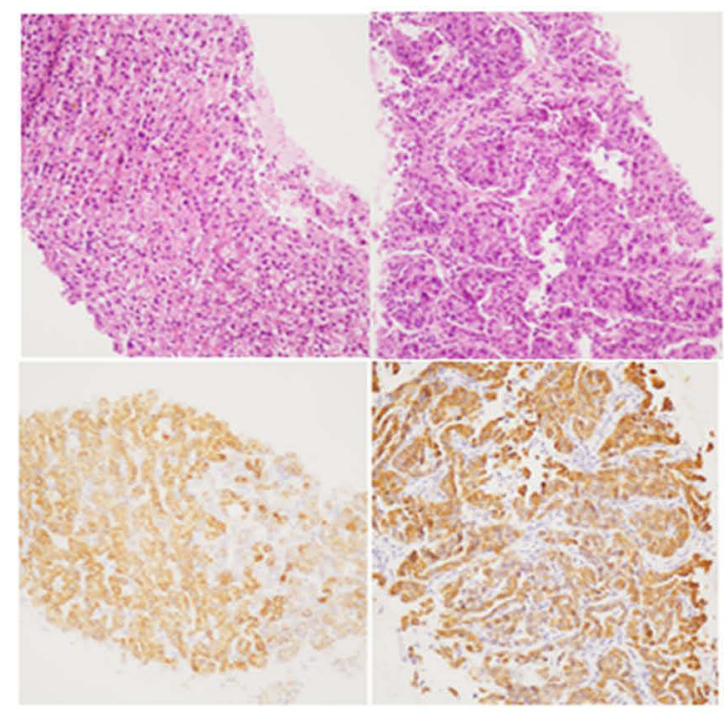

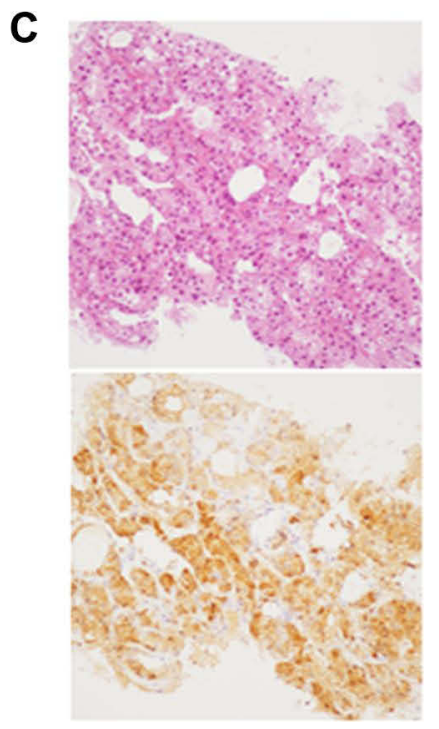

Figure I Three times of liver biopsy and the main immunohistochemical indexes. (A) On November 0I, 20I7, the patient presented with intrahepatic cholangiocarcinoma with low differentiation; the immunohistochemical results showed muc-I (+), GPC-3 (-), Hepa (-), CK7 (+), CKI9 (+), AFP (-), Ki67 (70\%+). (B) Postoperative pathology showed the combined HCC and ICC (cHCC-ICC), moderately differentiated; the immunohistochemical results showed muc-I (scattered + ), GPC-3 (oven + ), CK7 (+), CKI9 (+), AFP (-), Ki67 (30\%+). (C) Postoperative pathology showed HCC, moderately differentiated; the immunohistochemical results showed GPC-3 (part weak+), mucI (-), CK7 (part +), CKI9 (-), CD34 + (blood vessels+), CDI0 (+), Ki67 (<5\%+), AFP (-). 
GPC-3 (+), tumor mutation load (TML) was 20.1 Muts/Mb (TMB-H) with no other significant mutations. After operation, the liver lesions were completely ablated (Supplementary Figure 4A), but the patient developed liver abscess, which was recovered after more than 2 months of treatment including anti-inflammatory and drainage, the tumor was completely necrotic, and AFP was $177.1 \mathrm{ng} / \mathrm{mL}$.

In February 2019, the abdominal enhancement MRI showed the primary ICC nodules was disappeared (Supplementary Figure 4B), and 2 nodules were found in right lobe of liver and considered as active focus (Figure 2A), AFP level increased to $203 \mathrm{ng} / \mathrm{mL}$ again, and the patient was continue given 5 cycles of bi-specific antibody conjugated CIK (GPC-3) treatment (Figure 2C). In December 2019, liver lesions were significantly enlarged, new lesions appeared, AFP was $4553 \mathrm{ng} / \mathrm{mL}$, and the efficacy was assessed as PD (Figure 2A).

On December 19, 2019, the patient was performed with liver biopsy again, postoperative pathology showed HCC, moderately differentiated (Figure 1C). Genetic testing showed no significant mutations. The patient received TACE on December 2, 2019, and was subsequently not re-examined and treated due to COVID-19. In April 2020, abdominal MRI showed that the progress of intrahepatic tumor was obvious, AFP level was 20,550 ng/mL. From April 26, 2020, the patient received anti-PD-1 antibody therapy (Tislelizumab intravenous injection $200 \mathrm{mg}$, once every 3 weeks) for 10 cycles. On July 16, 2020 (4 cycles), the efficacy was assessed as PR, AFP level decreased to $8.7 \mathrm{ng} / \mathrm{mL}$. In October 2020, the efficacy was assessed as CR (Figure 2B and D). At present, patients still receive Tislelizumab immunotherapy and with good tolerance. The case treatment summary is shown in Supplementary Figure 5.

\section{Clinical Case Two}

A 65-year-old female, who had medical history of hypertension and type 2 diabetes, was diagnosed with HCC in local hospital in July 2016 and underwent radiofrequency ablation. In September 2017, she visited our hospital, the enhanced abdominal MRI showed multiple active liver lesions. On September 12, 2017, the patient was performed with microwave ablation, until lesions completely. In September 2018, the enhanced abdominal MRI showed multiple new active lesions in the liver, AFP was $806.2 \mathrm{ng} /$ $\mathrm{mL}$, Child-Pugh grade was 5 points. On September 11, 2018, she underwent microwave ablation and liver biopsy, the postoperative pathological results showed that moderately differentiated of HCC. Immunohistochemistry showed GPC-3 (+), CK7 (-), CK19 (-), CD34 (vascular+) and MUC1 (-) (Figure 3A). Finally, she was confirmed with primary HCC, Hepatitis B cirrhosis, hypertension, type 2 diabetes.

From October 2018 to March 2020, she was enrolled in the clinical trial "A Randomized, Controlled Phase II Clinical Study of Activated CIK Armed with Bispecific Antibody for

\section{A}
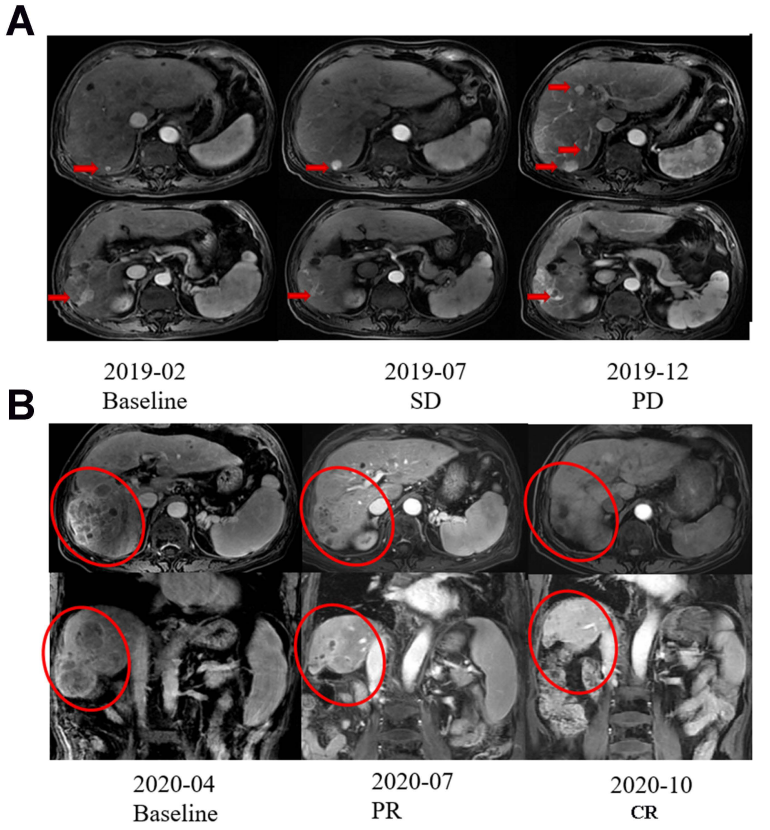

C
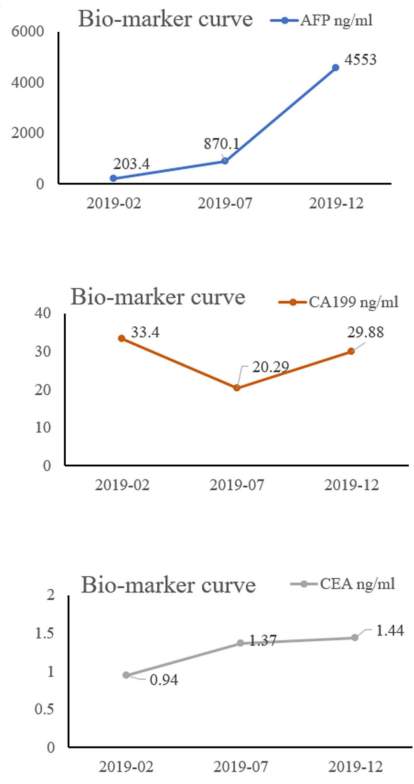

D
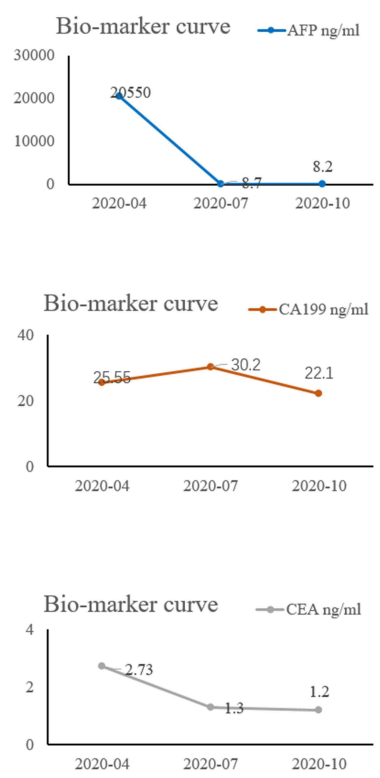

Figure 2 Changes of $(\mathbf{A})$ right lobe lesions and (C) tumor markers during bi-specific antibody conjugated CIK treatment from February to December 20 I9. (B) The efficacy was assessed as CR after the patient injected with PD-I and (D) Biomarker curve from April to October 2020. 
A
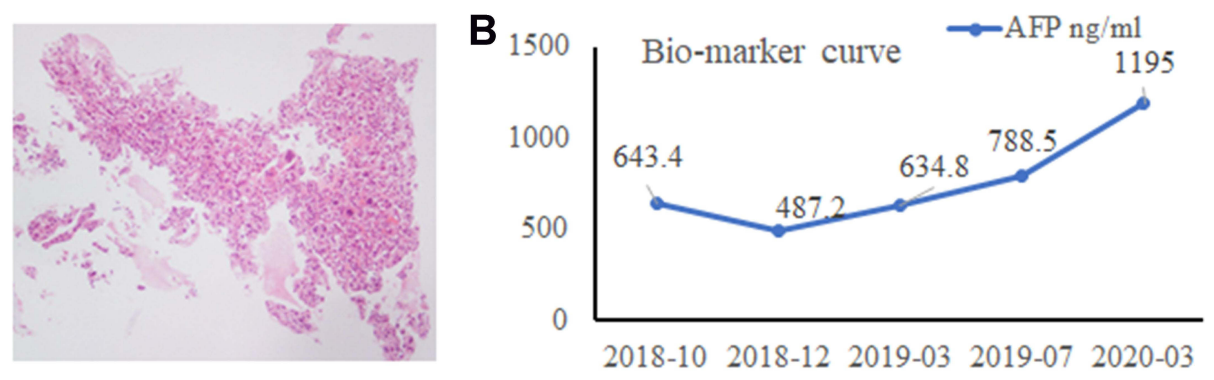

C

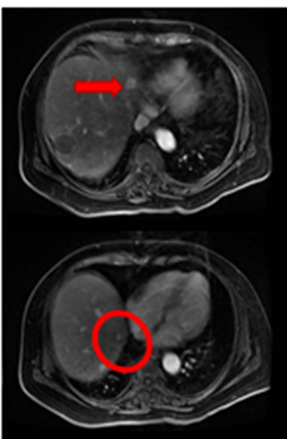

2018-10

Baseline

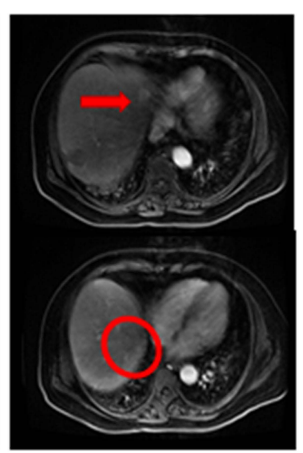

2019-09

SD

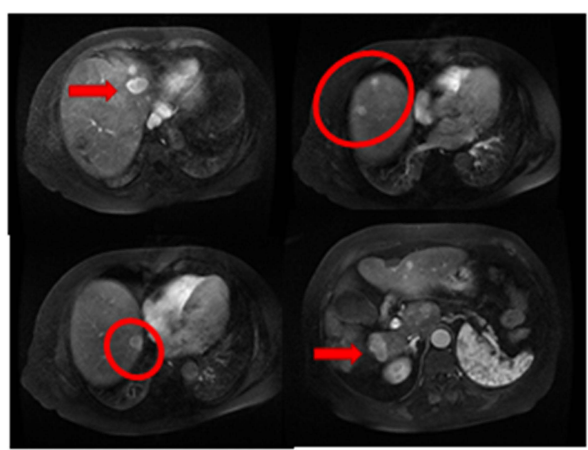

2020-03

PD

Figure 3 (A) The pathological results showed that moderately differentiated of HCC. (B) Changes of AFP and (C) imaging before and after bi-specific antibody conjugated $\mathrm{CIK}$ treatment from October 2018 to March 2020. Red arrows pointed to target lesions and red circles marked non-target lesions.

Advanced Liver Cancer" (NTC03146637), and were treated with bi-specific antibody conjugated CIK Cells (GPC-3) for 4 +4 cycles (Supplementary Figure 2). During this period, according to RECIST or modified RECIST, the efficacy was SD. In March 2020, the patient discontinued CIK therapy due to the disease progressed (Figure 3B and C), and was respectively performed with interventional therapy in March and June 2020. In August 2020, the enhanced abdominal MRI showed multiple active liver lesions, some of which were slightly larger than before, and some of which were new. AFP level was $911.4 \mathrm{ng} / \mathrm{mL}$. The efficacy was assessed as
PD. From August 18, 2020, she received anti-PD-1 antibody therapy (Sintilimab intravenous injection $200 \mathrm{mg}$, once every 3 weeks).

In September 2020, the second cycle of PD-1 was suspended due to the occurrence of immune mumps, which improved after hormone shock therapy. On October 13, 2020 (After using 1 cycle of PD-1), the efficacy was assessed as PR, AFP level was reduced to $13.88 \mathrm{ng} / \mathrm{mL}$. On December 26, 2020 (After using 4 cycle of PD-1), the efficacy was assessed as CR, AFP level was $3.38 \mathrm{ng} / \mathrm{mL}$ (Figure $4 \mathrm{~A}$ and $\mathrm{B}$ ). At present, the patient is
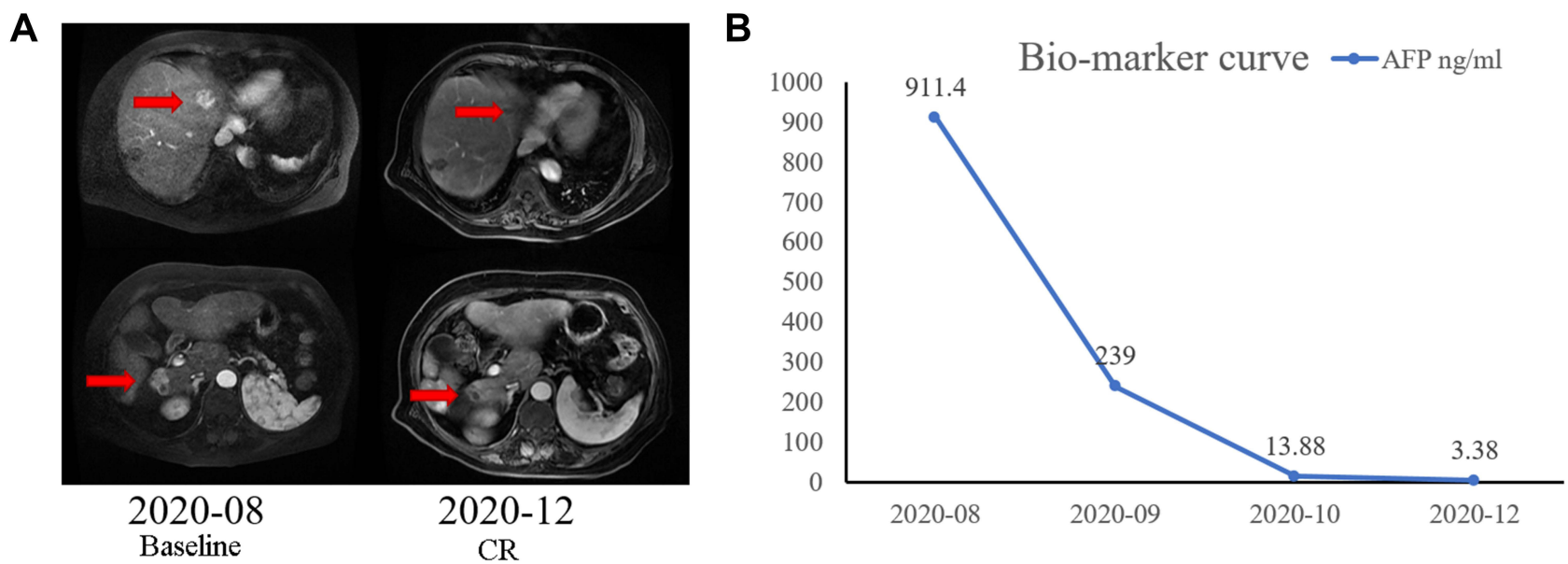

Figure 4 (A) Changes of imaging and (B) AFP before and after PD-I treatment from August to December 2020. 
still receiving sintilimab immunotherapy and with good tolerance.

\section{Discussion}

Recently, one meta-analysis has demonstrated the feasibility and effectiveness of CIK adoptive cell immunotherapy in HCC adjuvant, advanced or second-line therapy. ${ }^{8} \mathrm{CIK}$ therapy could improve the progression-free survival of HCC patients, but did not significantly improve the overall survival of patients, indicating CIK treatment could not benefit all HCC patients due to its non-specificity. Moreover, the specific recognition ability of CIK cells is limited, when CIK cells are diluted in vivo, they cannot be efficiently recruited to the tumor site. In this study, antibodies targeting the cancer-associated antigen Mucin 1 (MUC-1) or Glypican 3 (GPC-3) were chemically conjugated with $\mathrm{CD} 3$ antibodies that recognize $\mathrm{T}$ cells to form bi-specific antibody conjugated CIK cell to assist T cells in killing cancer cells. ${ }^{11}$

Two patients achieved CR with anti-PD-1 antibody therapy post-progression on bi-specific antibody conjugated CIK immunotherapy, indicating this therapeutic regime could get good and long-lasting clinical responses. The exact mechanism of the antitumor response to this treatment regimen is still unknown, we hypothesize the following several underlying factors may play important roles. Firstly, CIK therapy not only increases the number of $\mathrm{T}$ lymphocytes in tumor microenvironment but also provides cytokines for existing tumor-infiltrating $\mathrm{T}$ cells, enhances the anti-tumor function of $\mathrm{T}$ cells, which is conducive to anti-tumor response. ${ }^{12}$ Secondly, CIK cells may have synergistic effect with anti-PD-1 antibodies. It has reported reducing the expression of PD-1 in HCC cells could improve the sensitivity of CIK to HCC cells in nude mice and increase its anti-tumor activity. ${ }^{13}$ After co-culture of CIK cells with gastrointestinal tumor cells, the expression of PD-L1 on the surface of tumor cells or CIK cells were both up-regulated; blocking PD-1/PD-L1 pathway could not only block the immunosuppressive pathway, but also increase the expression of NKG2D, so improving the antitumor activity of CIK cells. ${ }^{14}$ Thirdly, CIK therapy may increase the number of some important anti-tumor immune cells in the body, improving the efficacy of anti-PD-1 therapy and reversing its resistance.

In these two patients, the percentage of $\mathrm{T}$ lymphocytes and $\mathrm{CD}^{+} \mathrm{T}$ cells increased, and the percentage of $\mathrm{CD}^{+} \mathrm{T}$ cells and the ratio of $\mathrm{CD}^{+} /$ $\mathrm{CD}^{+} \mathrm{T}$ cells decreased during CIK treatment (Supplementary Tables 1 and 2 ). $\mathrm{CD}^{+} \mathrm{T}$ cells are the direct effector cells to kill tumors, which can release granzyme and activate the Fas/FasL pathway to promote tumor cell apoptosis through the recognition of $\mathrm{T}$ lymphocyte antigen receptor and tumor antigen. In terms of killing cancer cells, the function of $\mathrm{CD}^{+}$ $\mathrm{T}$ cells is more important than that of $\mathrm{CD} 4^{+} \mathrm{T}$ cells. ${ }^{15}$ One study focused on CIK combined with PD-1 for treatment of NSCLC have shown the combination therapy could increase $\mathrm{CD}^{+} \mathrm{CD} 16^{+} \mathrm{CD} 56^{+} \mathrm{T}$ cells, which play central roles in innate anti-tumor immune regulation, thus to reverse the drug resistance of anti-PD-1 treatment and enhance the clinical response of tumor patients. ${ }^{11}$ After CIK treatment, the proportion of $\mathrm{CD}^{+}$ $\mathrm{T}$ cells increased. On this basis, PD-1 antibody was used to block the inhibition signal of $\mathrm{T}$ cells, which may be one of the reasons for CIK sequential PD-1 treatment to achieve better curative effect.

In case two, the percentage of $\mathrm{CD}^{+} \mathrm{T}$ cells decreased to the baseline value after CIK treatment, while increased during the subsequent PD-1 treatment, this change suggested that it may be related to the efficacy. Data from the Cancer Genome Atlas (TCGA) HCC cohort suggested that intratumoral $\mathrm{CD}^{+} \mathrm{T}$ cell infiltration was associated with better prognosis. ${ }^{16}$ Macek Jilkova et al found that a lower baseline intratumoral $\mathrm{CD}^{+} / \mathrm{CD}^{+} \mathrm{T}$ cell ratio was associated with better survival and prognosis of HCC patients. ${ }^{17}$ Higher baseline intratumoral PD-1 CD8 T cells were associated with better efficacy of PD-1/PD-L1 inhibitors. Further, Kamphorst et al found among non-small cell lung cancer (NSCLC) patients who received first and second courses of treatment PD- 1 inhibitors, $\mathrm{Ki} 67^{+} \mathrm{PD}-1^{+} \mathrm{CD} 8$ $\mathrm{T}$ cells increased in approximately $70 \%$ patients, PD$1^{+} \mathrm{CD} 8 \mathrm{~T}$ cell response was delayed or disappeared in $70 \%$ disease progression patients, and PD- $1^{+} \mathrm{CD} 8 \mathrm{~T}$ cell response occurred in $80 \%$ clinical benefit patients within 4 weeks of treatment. ${ }^{18}$ In summary, it could speculate that in HCC patients, CIK therapy may increase the proportion of certain immune cells, such as $\mathrm{CD}^{+} \mathrm{T}$ cells, thus enhance the efficacy of anti-PD-1 therapy. Due to the small number of cases, the exact mechanism of these aspects needs to be further studied.

In addition, liver biopsy was performed in case one at three different stages of the disease course, and the pathological results were ICC, cHCC-ICC and primary HCC, 
respectively. When HCC was diagnosed in October 2017, pathological, imaging and laboratory results all supported ICC. In October 2018, AFP was significantly elevated when the disease was progressing, and CA199 was within the normal range. Moreover, two tissues were taken from the tumor at different sites during the second liver biopsy, one indicating $\mathrm{HCC}$ and the other indicating ICC, rather than $\mathrm{cHCC}-\mathrm{ICC}$, indicating that HCC was new lesion. In December 2019, the APF level increased to $20,550 \mathrm{ng} / \mathrm{mL}$, combined with the third pathological and imaging findings, it was consistent with HCC, indicating that ICC had been cured at this time. That was to say, the subtypes of HCC dynamically changed in patients during treatment.

\section{Conclusion}

In summary, anti-PD-1 antibody therapy after prior progression on bi-specific antibody conjugated CIK immunotherapy may be efficacy and safety for HCC patients. This may provide a new idea for treatment of $\mathrm{HCC}$ in the future.

\section{Data Sharing Statement}

The raw data supporting the conclusions of this article will be made available by the authors, without undue reservation.

\section{Ethics Approval and Informed Consent}

The study was approved by the ethics committee of The Fifth Medical Center of Chinese PLA General Hospital. Written informed consent was obtained from the individual(s) for the publication of any potentially identifiable images or data included in this article.

\section{Consent for Publication}

We obtained consent to publication of medical data.

\section{Author Contributions}

All authors made a significant contribution to the work reported, whether that is in the conception, study design, execution, acquisition of data, analysis and interpretation, or in all these areas; took part in drafting, revising or critically reviewing the article; gave final approval of the version to be published; have agreed on the journal to which the article has been submitted; and agree to be accountable for all aspects of the work.

\section{Funding}

This study was supported by the Science Technology and Innovation Committee of Shenzhen Municipality (No.2019N002) and Capital's Funds for Health Improvement and Research (No.Z181100001718075).

\section{Disclosure}

The authors declare that they have no conflict of interest.

\section{References}

1. Cui H, Dai G, Guan J. Programmed cell death protein-1 (PD-1)targeted immunotherapy for advanced hepatocellular carcinoma in real world. Onco Targets Ther. 2020;13:143-149. doi:10.2147/OTT. S234868

2. Finn RS, Qin S, Ikeda M, et al. Atezolizumab plus Bevacizumab in unresectable hepatocellular carcinoma. $N$ Engl J Med. 2020;382 (20):1894-1905. doi:10.1056/NEJMoa1915745

3. Finn RS, Ikeda M, Zhu AX, et al. Phase Ib study of Lenvatinib plus Pembrolizumab in patients with unresectable hepatocellular carcinoma. J Clin Oncol. 2020;38(26):2960-2970. doi:10.1200/ JCO.20.00808

4. Yau T, Kang YK, Kim TY, et al. Efficacy and safety of nivolumab plus Ipilimumab in patients with advanced hepatocellular carcinoma previously treated with Sorafenib: the CheckMate 040 randomized clinical trial. JAMA Oncol. 2020;6(11):e204564. doi:10.1001/ jamaoncol.2020.4564

5. Luo XY, Wu KM, He XX. Advances in drug development for hepatocellular carcinoma: clinical trials and potential therapeutic targets. J Exper Clin Cancer Res. 2021;40(1):172. doi:10.1186/ s13046-021-01968-w

6. Liu X, Yi Y. Recent updates on Sintilimab in solid tumor immunotherapy. Biomark Res. 2020;8(1):69. doi:10.1186/s40364020-00250-z

7. Cai XR, Li X, Lin JX, et al. Autologous transplantation of cytokine-induced killer cells as an adjuvant therapy for hepatocellular carcinoma in Asia: an update meta-analysis and systematic review. Oncotarget. 2017;8(19):31318-31328. doi:10.18632/ oncotarget. 15454

8. Cao J, Kong FH, Liu X, Wang XB. Immunotherapy with dendritic cells and cytokine-induced killer cells for hepatocellular carcinoma: a meta-analysis. World J Gastroenterol. 2019;25(27):3649-3663. doi:10.3748/wjg.v25.i27.3649

9. Hui Z, Zhang X, Ren B, Li R, Ren X. Rapid response of advanced squamous non-small cell lung cancer with Thrombocytopenia after first-line treatment with Pembrolizumab plus autologous cytokine-induced killer cells. Front Immunol. 2015;6:633. doi:10.3389/fimmu.2015.00633

10. Wang Z, Liu X, Till B, Sun M, Li X, Gao Q. Combination of cytokine-induced killer cells and programmed cell death-1 blockade works synergistically to enhance therapeutic efficacy in metastatic renal cell carcinoma and non-small cell lung cancer. Front Immunol. 2018;9:1513. doi:10.3389/fimmu.2018.01513

11. Zhengcheng LI, Chengsi HE, Xinjian YU, Haoyang YU. Production and function evaluation of a kind of bispecific antibody crosslinked with PLGA nanoparticles in vitro. Immunol J. 2016;32(1):34-37.

12. Yu B, Wang J, He C, et al. Cytokine-induced killer cell therapy for modulating regulatory T cells in patients with non-small cell lung cancer. Exp Ther Med. 2017;14(1):831-840. doi:10.3892/etm.2017.4562

13. Huang F, Wang B, Zeng J, Sang S, Lei J, Lu Y. MicroRNA-374b inhibits liver cancer progression via down regulating programmed cell death-1 expression on cytokine-induced killer cells. Oncol Lett. 2018;15(4):4797-4804. doi:10.3892/ol.2018.7951 
14. Dai C, Lin F, Geng R, et al. Implication of combined PD-L1/PD-1 blockade with cytokine-induced killer cells as a synergistic immunotherapy for gastrointestinal cancer. Oncotarget. 2016;7 (9):10332-10344. doi:10.18632/oncotarget.7243

15. Farhood B, Najafi M, Mortezaee K. CD8(+) cytotoxic T lymphocytes in cancer immunotherapy: a review. J Cell Physiol. 2019;234 (6):8509-8521. doi:10.1002/jcp.27782

16. Zhang W, Song Z, Xiao J, et al. Blocking the PD-1/PD-L1 axis in dendritic cell-stimulated cytokine-induced killer cells with pembrolizumab enhances their therapeutic effects against hepatocellular carcinoma. $J$ Cancer. 2019;10(11):2578-2587. doi:10.7150/jca.26961
17. Macek Jilkova Z, Aspord C, Kurma K, et al. Immunologic features of patients with advanced hepatocellular carcinoma before and during Sorafenib or anti-programmed death-1/programmed death-L1 treatment. Clin Transl Gastroenterol. 2019;10(7):e00058. doi:10.14309/ctg.0000000000000058

18. Kamphorst AO, Pillai RN, Yang S, et al. Proliferation of PD-1+ CD8 $\mathrm{T}$ cells in peripheral blood after PD-1-targeted therapy in lung cancer patients. Proc Natl Acad Sci U S A. 2017;114(19):4993-4998. doi:10.1073/pnas.1705327114

\section{Publish your work in this journal}

OncoTargets and Therapy is an international, peer-reviewed, open access journal focusing on the pathological basis of all cancers, potential targets for therapy and treatment protocols employed to improve the management of cancer patients. The journal also focuses on the impact of management programs and new therapeutic

Submit your manuscript here: https://www.dovepress.com/oncotargets-and-therapy-journal agents and protocols on patient perspectives such as quality of life, adherence and satisfaction. The manuscript management system is completely online and includes a very quick and fair peer-review system, which is all easy to use. Visit http://www.dovepress.com/ testimonials.php to read real quotes from published authors. 